\title{
Development of captive breeding and seed production techniques for Giant River Catfish Sperata seenghala
}

\begin{abstract}
The Giant River Catfish Sperata seenghala, locally known as "Guizza Air" (and hereafter referred to as just Guizza), has significant aquacultural and conservational values, but closer monitoring of the fish is now badly needed. To protect the species from extinction, an experiment was undertaken to breed and produce seed of Guizza in a captive-rearing system. In our study, Guizza were successfully bred and the naturally produced fry were reared at different stocking densities in nine earthen nursery ponds having an area of 0.012 ha each with an average depth of $0.8 \mathrm{~m}$. Three stocking densities were evaluated in three replicates each. Fry produced from natural propagation that were stocked at 100,000/ha were defined as treatment 1 (T1), those stocked at 150,000/ha as treatment 2 (T2), and those stocked at 200,000/ha as treatment 3 (T3). At stocking, all reared fry were of the same age-group with a mean \pm SD length and weight of $1.74 \pm 0.27 \mathrm{~cm}$ and $0.20 \pm 0.03 \mathrm{~g}$, respectively. Fry in all the experimental ponds were reared with commercial Saudi Bangla (SABINCO) nursery feed (32.06\% crude protein) for the first $14 \mathrm{~d}$ and starter-I (31.53\% crude protein) for days 15-56. Growth (final weight, final length, weight gain, length gain, and specific growth rate) and survival of the fingerlings were significantly higher in T1 than in T2 and T3. Feed conversion rate was significantly lower in T1 than in T2 and T3. Moreover, the physicochemical measurements and plankton population of the pond water were within the optimal level for fish culture, with plankton abundance being higher in T1 than in T2 and T3. Consistently higher net benefits were obtained from T1 than from T2 and T3. Hence, of the three stocking densities, 100,000 fry/ha was the most suitable density for rearing Guizza fingerlings in nursery ponds.
\end{abstract}

Keyword: Sperata seenghala; Guizza; Stocking densities; Captive breeding; Seed production 